\title{
RETRACTIONS AND QUASI-MONOTONE MAPPINGS OF UNICOHERENT SPACES
}

\author{
M. M. MCWATERS AND J. H. REED
}

\begin{abstract}
It is shown that retractions of connected, locally connected, unicoherent spaces are unieoherent, and that quasimonotone maps preserve the unicoherence of any connected unicoherent space.
\end{abstract}

1. Introduction. It is well known [3] that the unicoherence of locally connected metrizable continua is invariant under maps which are either (i) interior, (ii) monotone or (iii) retractions. Wallace [2] showed that quasi-monotone maps preserve the unicoherence of such continua, and recently Charatonik [1] proved that confluent maps preserve the unicoherence of these continua. Charatonik also showed that the class of confluent maps includes the interior maps as well as the monotone maps on continua, and that quasi-monotone and confluent maps coincide on locally connected continua. It should be remarked that a monotone map preserves the unicoherence of, and is quasi-monotone on, any continuum.

Our purpose here is to show that the conditions of compactness and metrizability may be dropped in the first mentioned theorem for retractions; and that quasi-monotone maps preserve the unicoherence of any connected space. We give examples to show that in the absence of compactness neither monotone, confluent, nor interior maps preserve unicoherence.

We assume throughout the paper that the spaces under discussion are Hausdorff, and use the asterisk to denote the closure of a set.

2. Retractions of connected, locally connected, unicoherent spaces. The following two results enable us to show that such retractions preserve unicoherence.

LEMMA 2.1. Let $X$ be a locally connected, connected space and let $C, D$ be closed connected subsets of $X$ with $X=C \cup D$. If $C \cap D=A \cup B$ where $A$ and $B$ are closed disjoint subsets of $C \cap D$ then some component of $D-$ $(A \cup B)$ has limit points in both $A$ and $B$.

\footnotetext{
Received by the editors April 30, 1971.

AMS 1970 subject classifications. Primary 54C15, 54F55; Secondary 54C10, 54D05. Key words and phrases. Retraction, unicoherent, quasi-monotone map, confluent map. ${ }^{1}$ This work was supported in part by the University of South Florida Research Council.
}

(c) American Mathematical Society 1972 
Proof. Suppose no component of $D-(A \cup B)$ has limit points in both $A$ and $B$. Let $R(A)$ be the union of all components of $D-(A \cup B)$ which have limit points in $A$, and let $R(B)$ be the union of all components of $D-(A \cup B)$ which have limit points in $B$. Let $S(A)=A \cup R(A)$ and $S(B)=$ $B \cup R(B)$.

Since $D-(A \cup B)$ is an open subset of $X, D-(A \cup B)$ is locally connected. Thus each component of $D-(A \cup B)$ is open in $D$, and therefore no component of $D-(A \cup B)$ is closed in $D$ since $D$ is connected. Thus each component of $D-(A \cup B)$ has limit points in either $A$ or $B$, and hence $S(A) \cup S(B)=D$.

Since no component of $D-(A \cup B)$ has limit points in both $A$ and $B$, we have $S(A) \cap S(B)=\varnothing$. Thus since $D$ is connected, $\left[S(A) \cap S(B)^{*}\right] \cup$ $\left[S(A)^{*} \cap S(B)\right] \neq \varnothing$. Assume $S(A) \cap S(B)^{*} \neq \varnothing$ and let $x \in S(A) \cap S(B)^{*}$. We consider two cases, either $x \in A$ or $x \notin A$.

If $x \in A$, let $U$ be an open connected subset of $X$ such that $x \in U$ and $U \cap B=\varnothing$. Let $V=U \cap D$. Then $V \cap S(B) \neq \varnothing$ but $V \cap B=\varnothing$. Thus there exists a component $K$ of $D-(A \cup B)$ with limit points in $B$ such that $V \cap K \neq \varnothing$. Since $K^{*} \cap A=\varnothing, K \cup B$ is closed in $D$ and $X$. Therefore, $V \cap K=(U \cap D) \cap K=U \cap(K \cap D)=U \cap K=U \cap(K \cup B)$ is closed in $U$. But $K$ is a component of the open subset $D-(A \cup B)$ of a locally connected space, hence $K$ is open in $X$. Thus $K \cap U=V \cap K$ is also open in $U$. Since $U$ is connected, $U=V \cap K$. Thus $x \in V \cap K$, and $x \in A$, hence $K \cap A \neq \varnothing$ which contradicts $K^{*} \cap A=\varnothing$.

If $x \notin A$, then $x \in R(A)$, hence there exists a component $K_{1}$ of $D-(A \cup B)$ which has limit points in $A$ such that $x \in K_{1}$. Thus $K_{1}^{*} \cap B=\varnothing$ and so $x \notin A \cup$ $B$. Therefore there exists an open connected subset $U$ of $X$ such that $x \in U \subseteq D-(A \cup B)$. But since $x$ is a limit point of $S(B), U$ must contain some point $y \in R(B)$, where $y$ is an element of some component $K_{2}$ of $D-(A \cup B)$ which has limit points in $B$. Thus $x \in K_{1} \cap U$ and $y \in K_{2} \cap U$ which means $U \subseteq K_{1}$ and $U \subseteq K_{2}$. Therefore $K_{1}=K_{2}$, and $K_{1}$ has limit points in each of $A$ and $B$, a contradiction.

TheOREM 2.2 (WILDER [4, Theorem 4.13, p. 51]). If $X$ is a connected, locally connected space which is unicoherent, then for any closed subset $M$ of $X$ and components $C, D$ of $M, S-M$ respectively, the set $C \cap \operatorname{Bd}(D)$ is connected.

THEOREM 2.3. Let $X$ be a connected, locally connected, unicoherent space, and let $f$ be a retraction on $X$. Then $f(X)$ is unicoherent.

Proof. Suppose $f(X)$ is not unicoherent. Then $f(X)$ is a closed, connected, locally connected subset of $X$ which can be expressed as the union of two closed connected subsets $C \cup D$ such that $C \cap D$ is not 
connected. Let $C \cap D=A \cup B$ where $A$ and $B$ are disjoint closed subsets of $f(X)$. Let $M=f^{-1}(C)$ and let $Q$ be the component of $f^{-1}(C)$ containing $C$. Consider $X-M=f^{-1}(D-C)$. By Lemma 2.1, there exists a component $K$ of $D-C$ having limit points in each of $A$ and $B$. Since $K \subseteq D-C \subseteq f^{-1}(D-C)=X-M$, there exists a component $K_{1}$ of $X-M$ having limit points in each of $A$ and $B$, and by Theorem 2.2, $Q \cap \operatorname{Bd}\left(K_{1}\right)$ is connected.

We show that $f\left[Q \cap \operatorname{Bd}\left(K_{1}\right)\right] \subseteq C \cap D$. Let $x \in Q \cap \operatorname{Bd}\left(K_{1}\right)$. Then $f(x) \in C$. Also, since $K_{1} \subseteq f^{-1}(D-C)$ we have $K_{1}^{*} \subseteq\left[f^{-1}(D-C)\right]^{*} \subseteq f^{-1}\left[(D-C)^{*}\right] \subseteq$ $f^{-1}\left(D^{*}\right)=f^{-1}(D)$. Thus if $x \in \operatorname{Bd}\left(K_{1}\right)$ then $f(x) \in D$. Hence $f\left[Q \cap \operatorname{Bd}\left(K_{1}\right)\right] \subseteq$ $C \cap D$.

Since $K \subseteq K_{1}$ and $K^{*} \cap A \neq \varnothing \neq K^{*} \cap B$, we have $K_{1}^{*} \cap A \neq \varnothing \neq K_{1}^{*} \cap B$. Also, $A \subseteq f^{-1}(C)$ and $B \subseteq f^{-1}(C)$, and since $K_{1} \subseteq f^{-1}(D-C)$ we have $K_{1} \cap$ $A=\varnothing=K_{1} \cap B$. Moreover, $K_{1}$ is an open subset of $X$ since $K_{1}$ is a component of the open set $f^{-1}(D-C)$ in $X$, and so $\operatorname{Bd}\left(K_{1}\right)=K_{1}^{*}-K_{1}$. Thus $\operatorname{Bd}\left(K_{1}\right) \cap A \neq \varnothing$ and $\operatorname{Bd}\left(K_{1}\right) \cap B \neq \varnothing$. But since $C \subseteq Q$, we have $\operatorname{Bd}\left(K_{1}\right) \cap$ $(A \cup B) \subseteq \operatorname{Bd}\left(K_{1}\right) \cap C \subseteq \operatorname{Bd}\left(K_{1}\right) \cap Q$. Thus $\left[\operatorname{Bd}\left(K_{1}\right) \cap A\right] \cup\left[\operatorname{Bd}\left(K_{1}\right) \cap B\right]=$ $f\left[\operatorname{Bd}\left(K_{1}\right) \cap(A \cup B)\right] \subseteq f\left[\operatorname{Bd}\left(K_{1}\right) \cap C\right] \subseteq f\left[\operatorname{Bd}\left(K_{1}\right) \cap Q\right]$. Therefore,

$$
f\left[\operatorname{Bd}\left(K_{1}\right) \cap Q\right] \cap A \neq \varnothing \text { and } f\left[\operatorname{Bd}\left(K_{1}\right) \cap Q\right] \cap B \neq \varnothing .
$$

But then $f\left[Q \cap \operatorname{Bd}\left(K_{1}\right)\right]$ is not connected, a contradiction.

3. Quasi-monotone maps on connected unicoherent spaces. We begin with the following definitions.

Definition. A map $f$ of a space $X$ onto a space $Y$ is quasi-monotone if for each closed, connected subset $Q$ of $Y$ with a nonempty interior, the set of components of $f^{-1}(Q)$ is finite and $f$ maps each component onto $Q$.

DEFINITION. Let $\mathscr{S}$ be a collection of subsets of a space $X$. A chain in $\mathscr{S}$ is a finite sequence $X_{1}, X_{2}, \cdots, X_{k}$ of elements of $\mathscr{S}$ such that $X_{i} \cap X_{j} \neq$ $\varnothing$ if and only if $|i-j| \leqq 1$.

THEOREM 3.1. Let $X$ be a connected, unicoherent space and let $f$ be a quasi-monotone map of $X$ onto $Y$. Then $Y$ is unicoherent.

Proof. Let $C$ and $D$ be closed connected proper subsets of $Y$ such that $Y=C \cup D$. We show that $C \cap D$ is connected.

Since $f$ is quasi-monotone, $f^{-1}(C)$ has a finite number of components, say $K_{1}, K_{2}, \cdots, K_{n}$. Similarly $f^{-1}(D)$ has a finite number of components, say $L_{1}, L_{2}, \cdots, L_{m}$. Each $K_{i}$ intersects some $L_{j}$ and each $L_{i}$ intersects some $K_{j}$, otherwise $X$ is not connected. Also, $f\left(K_{i} \cap f^{-1}(D)\right)=f\left(L_{j} \cap f^{-1}(C)\right)=$ $C \cap D$ for each $K_{i}$ and $L_{j}$.

Consider $K_{1}$. Reindex the $L$ 's so that $L_{1}, L_{2}, \cdots, L_{p}$ each intersect $K_{1}$ and $L_{p+1}, L_{p+2}, \cdots, L_{m}$ each do not intersect $K_{1}$. Let $\mathscr{A}=\left\{K_{1}, \cdots, K_{n}\right.$, 
$\left.L_{1}, \cdots, L_{m}\right\}$ and let $\mathscr{B}=\mathscr{A}-\left\{K_{1}\right\}$. For each $i, 1 \leqq i \leqq p$, let $M_{i}=\{P \mid P \in \mathscr{B}$, $P$ can be joined to $L_{i}$ by a chain in $\left.\mathscr{B}\right\}$, and let $L_{i}^{\#}=\bigcup\left\{Q \mid Q \in M_{i}\right\}$.

Each $P \in \mathscr{B}$ is a member of some $M_{i}, 1 \leqq i \leqq p$. For if $P \notin M_{i}$ for any $i$, let $\mathscr{C}=\{A \in \mathscr{B}, A$ is a link of some chain in $\mathscr{B}$ starting at $P\}$. Let $\mathscr{D}=\mathscr{A}-\mathscr{C}$. Then $\mathscr{A}-\mathscr{C} \neq \varnothing$, since $L_{1}, L_{2}, \cdots, L_{p}$ and $K_{1}$ are in $\mathscr{A}-\mathscr{C}$. Also, $\mathscr{C}$ is nonempty, since $P \in \mathscr{C}$. Moreover, $K_{1}$ intersects no element of $\mathscr{C}$, and if an element of $\mathscr{A}-\mathscr{C}$ different from $K_{1}$ intersects an element of $\mathscr{C}$, then it is $\mathscr{C}$. Thus $(\bigcup \mathscr{C}) \cap(\bigcup(\mathscr{A}-\mathscr{C}))=\varnothing$ and $(\bigcup \mathscr{C}) \cup(\cup(\mathscr{A}-\mathscr{C}))=X$, a contradiction since $X$ is connected.

We consider two cases, depending upon how the $K$ 's and $L$ 's intersect.

Case 1. Suppose there exist $K_{i}$ for some $i$ such that $K_{i}$ intersects exactly one $L_{j}$. Reindex the $K$ 's and $L$ 's so that $K_{1} \cap L_{1} \neq \varnothing$ and $K_{1} \cap L_{j}=\varnothing$ for $2 \leqq j \leqq m$. We know each $P \in \mathscr{B}$ can be joined to $L_{1}$ by a chain in $\mathscr{B}$; i.e., $P \in M_{1}$. Thus $L_{1}^{\#}$ is closed and connected, and $K_{1} \cap L_{1}^{\#}=K_{1} \cap f^{-1}(D)$. Since $K_{1} \cup L_{1}^{\#}=X$, we have $K_{1} \cap f^{-1}(D)=K_{1} \cap L_{1}^{\#}$ is connected and hence $f\left(K_{1} \cap f^{-1}(D)\right)=C \cap D$ is connected. A similar argument shows that $C \cap D$ is connected if there exists $L_{i}$ for some $i$ such that $L_{i}$ intersects exactly one $K_{j}$.

Case 2. Suppose each $K_{i}$ intersects at least two $L_{j}$ 's and each $L_{i}$ intersects at least two $K_{j}$ 's. Consider $K_{1}$. Then $L_{1}, \cdots, L_{p}$ each intersect $K_{1}$ and $L_{p+1}, \cdots, L_{m}$ each do not intersect $K_{1}$ where $p \geqq 2$.

If there exist indices $q \neq s$ such that $L_{q}^{\#} \cap L_{s}^{\#} \neq \varnothing, 1 \leqq q$, $s \leqq p$, let $A=$ $\bigcup\left\{L_{i}^{\#} \mid L_{i}^{\#} \cap L_{a}^{\#} \neq \varnothing, 1 \leqq i \leqq p\right\}$. Then $A$ is closed and connected. Also $A \cap K_{1}$ is not connected, since $L_{Q} \subseteq A$ and $L_{s} \subseteq A$. Let

$$
B=K_{1} \cup\left(\bigcup\left\{L_{i}^{\#} \mid L_{i}^{\#} \cap L_{q}^{\#}=\varnothing, 1 \leqq i \leqq p\right\}\right) .
$$

Since each element of $\mathscr{B}$ is a member of some $M_{i}$ and therefore a subset of some $L_{i}^{\#}$, we have $A \cup B=X$. Also $B$ is connected, and $A \cap B=A \cap K_{1}$. Thus $X$ is not unicoherent, a contradiction.

If for all $q \neq s, 1 \leqq q, s \leqq p$, we have $L_{q}^{\#} \cap L_{s}^{\#}=\varnothing$, then consider $L_{\mathbf{1}}$. There exists $K_{\alpha(1)}$ in $\mathscr{B}$ such that $K_{\alpha(1)} \cap L_{1} \neq \varnothing$. Then there exists $L_{\beta(1)}$ in $\mathscr{B}-$ $\left\{L_{1}\right\}$ such that $L_{\beta(1)} \cap K_{\alpha(1)} \neq \varnothing$; there exists $K_{\alpha(2)}$ in $\mathscr{B}-\left\{K_{\alpha(1)}\right\}$ such that $K_{\alpha(2)} \cap L_{\beta(1)} \neq \varnothing$; and there exists $L_{\beta(2)}$ in $\mathscr{B}-\left\{L_{\beta(1)}\right\}$ such that $L_{\beta(2)} \cap$ $K_{\alpha(2)} \neq \varnothing$. In general, choose $K_{\alpha(n)}$ in $\mathscr{B}-\left\{K_{\alpha(n-1)}\right\}$ such that $K_{\alpha(n)} \cap$ $L_{\beta(n-1)} \neq \varnothing$, and choose $L_{\beta(n)}$ in $\mathscr{B}-\left\{L_{\beta(n-1)}\right\}$ such that $L_{\beta(n)} \cap K_{x(n)} \neq \varnothing$. Thus we construct the two sequences $K_{\alpha(1)}, K_{\alpha(2)}, \cdots$ and $L_{\beta(1)}, L_{\beta(2)}, \cdots$. Since there are only a finite number of $K$ 's and $L$ 's, some $K_{\alpha(i)}=K_{\alpha(j)}$, $i \neq j$, and some $L_{\beta(r)}=L_{\beta(t)}, r \neq t$. Let $u$ be the smallest integer such that for some $v<u, K_{\alpha(v)}=K_{\alpha(u)}$ or $L_{\beta(v)}=L_{\beta(u)}$. Suppose $K_{\alpha(v)}=K_{\alpha(u)}$. Then $|v-u| \geqq 2$ and $K_{\alpha(v)} \cap L_{\beta(v)} \neq \varnothing$ and $K_{\alpha(u)} \cap L_{\beta(u-1)} \neq \varnothing$. Also, $L_{\beta(v)} \neq$ $L_{\beta(u-1)}$. Now reindex the $K$ 's and $L$ 's so that $K_{1}=K_{\alpha(v)}=K_{\alpha(u)}$, and so that $L_{1}, \cdots, L_{p}$ intersect $K_{1}$ and $L_{p+1}, \cdots, L_{m}$ do not intersect $K_{1}$. Then for 
some $g, h$ such that $g \neq h, 1 \leqq g, h \leqq p$, we have $L_{\beta(v)}=L_{g}$ and $L_{\beta(u-1)}=L_{h}$. Then $L_{h}^{\#} \cap L_{0}^{\#} \neq \varnothing$, and as shown above, this leads to $X$ not unicoherent, a contradiction.

4. Some examples. A map $f$ of a space $X$ onto a space $Y$ is confluent if for each closed, connected subset $Q$ of $Y$, each component of $f^{-1}(Q)$ is mapped by $f$ onto $Q$. We show here that for noncompact, locally connected, connected spaces, a mapping $f$ may fail to preserve unicoherence when $f$ is interior, monotone or confluent.

EXAMPLE. Let $X$ be the graph of the function $\rho=\left(2+e^{\theta}\right) /\left(1+e^{\theta}\right)$, $-\infty<\theta<\infty$, in a polar coordinate system. Let $C$ be the unit circle, and let $f: X \rightarrow C$ be the function which maps each point $(\rho, \theta)$ in $X$ onto the point $(1, \theta)$ in $C$. Then $f$ is a confluent, interior map of $X$ onto $C$ and $C$ is not unicoherent.

The map $f$ of this example is not monotone, but the exampie of a one-toone mapping of a half-open interval onto a simple closed curve shows that monotone maps do not preserve unicoherence.

\section{REFERENCES}

1. J. J. Charatonik, Confluent mappings and unicoherence of continua, Fund. Math. 56 (1964), 213-220. MR 31 \#723.

2. A. D. Wallace, Quasi-monotone transformations, Duke Math. J. 7 (1940), 136-145. MR 2, 179.

3. G. T. Whyburn, Analytic topology, Amer. Math. Soc. Colloq. Publ., vol. 28, Amer. Math. Soc., Providence, R.I., 1942. MR 4, 86.

4. R. L. Wilder, Topology of manifolds, Amer. Math. Soc. Colloq. Publ., vol. 32, Amer. Math. Soc., Providence, R.I., 1963. MR 32 \#440.

Department of Mathematics, University of South Florida, Tampa, Florida 33620 